\title{
PROFISSIONALIZAÇÃO E REGULAÇÃO DA ATIVIDADE DO AGENTE COMUNITÁRIO DE SAÚDE NO CONTEXTO DA REFORMA SANITÁRIA
}

\author{
PROFESSIONALIZATION AND REGULATION OF THE COMMUNITY HEALTH \\ AGENT'S ACTIVITY IN THE CONTEXT OF THE SANITARY REFORM
}

Maria Helena Magalhães de Mendonça ${ }^{1}$

Resumo O artigo tem como tema a profissionalização da atividade do agente comunitário de saúde, no contexto das reformas sociais formuladas no processo de redemocratização do Brasil nos anos 80 e 90, com ênfase na Reforma Sanitária brasileira. $\mathrm{O}$ argumento central é a existência de uma relação entre projetos de renovação das práticas sociais em saúde, como a reorganização da atenção básica de saúde a partir dos Programas de Agentes Comunitários de Saúde (Pacs) e Saúde da Família (PSF), e a emergência de nova categoria de trabalhador em saúde, que exige um esforço de profissionalização, entendida como educação profissional específica e regulação da atividade no mercado de trabalho em saúde. Primeiro, trabalha-se o contexto em que se definem políticas sociais de saúde como parte da constituição da cidadania social, que se baseia nos princípios da universalidade e da igualdade entre beneficiários. No segundo momento, trata-se da emergência desse novo agente, do seu papel no processo de mudança na produção da saúde e do papel do Estado na normalização de sua atuação e regulação no mercado de trabalho em saúde. Por fim, reflete-se sobre os desafios que a recente regulação da profissão traz para a formação profissional dos trabalhadores em saúde, cujo enfrentamento favorece a implementação da estratégia de Saúde da Família e auxilia na consolidação do processo de Reforma Social.

Palavras-chave Reforma Sanitária; atenção básica; saúde da família; profissionalização dos agentes de saúde.
Abstract The article deals with the professionalization of the community health agent's activity in the context of the social reforms formulated during Brazil's return to democracy in the 80s and 90s, with emphasis on the Brazilian sanitary reform. The central argument is that there is a relationship between the projects for the renovation of social practices in health - such as the reorganization of basic health care through the Programmes of Community Health Agents (Pacs) and Family Health (PSF) - and the emergence of a new category of health worker that requires specific professional training and regulation of the activity in the health labour market. First, we look at the context in which the health social policies were defined as part of the development of a social citizenship, based on the principles of universality and equality between beneficiaries. In a second stage, we deal with the emergence of these new agents, the role they play in the changes taking place in the production of health and the State's role in producing norms to regulate the activity and its participation in the health labour market. Finally, we reflect upon the challenges that the recent regulation of the profession has brought for the professional training of health workers, using as example the community health agent's profile, his/her perceptions about the Family Health, his/her investment on training and his/her work process, all elements that contribute to the implementation of the Family Health strategy and help to consolidate the process of sectorial reform (ESCOREL, Sarah, GIOVANELLA, Ligia, MENDONÇA, Maria Helena M., MAGALHÃES, Rosana e SENNA, Mônica C., 2002).

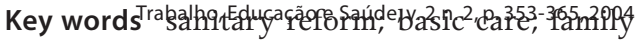
health; professionalization of health agents. 


\section{Reforma Sanitária e a reorganização da atenção básica}

A reorganização da atenção básica à saúde é objeto de debate no Brasil há pelo menos duas décadas e meia, quando surgem movimentos para ampliação do acesso da população aos serviços de saúde urbanos e para interiorização da assistência à saúde. Nessa fase, para o trabalho social, já se recrutavam na comunidade auxiliares de saúde, que, em parte, foram posteriormente absorvidos como auxiliares de enfermagem em postos de saúde, dentro de uma estratégia de profissionalização e de regulamentação do vínculo profissional. Não cabe aqui rever essa história já bem fundamentada em diversos trabalhos (Silva e Dalmaso, 2002; Merhy e Franco, s.d.), mas chamar atenção para o fato de que a profissionalização de uma nova atividade em saúde torna-se um fato social sempre que surgem projetos de renovação das práticas sociais.

Assim, parte-se da implementação de reformas sociais, com ênfase na Reforma Sanitária, durante os primeiros anos da década de 90. Consoante os princípios da seguridade social, política formulada no contexto de redemocratização do Estado brasileiro, a reestruturação da política social visa a compor um modelo de proteção social abrangente, justo, equânime e democrático, refazendo o campo da cidadania social. Este modelo tem como fim ampliar os direitos sociais concernentes a um Estado de Bem-Estar Social, com base na concepção de uma dívida social do Estado brasileiro a ser resgatada com a renovação das políticas sociais (Mendonça, 2002).

Contudo, ao ideal constitucional de um Estado de Bem-Estar Social contrapõe-se a resistência em regulamentar os direitos sociais ${ }^{2}$, que se prolonga até as revisões constitucionais de 1993-95, que ajustam os projetos reformistas ao pensamento neoliberal. Os debates políticos que levaram a uma reforma do Estado na contramão do Estado de bem-estar não paralisam, contudo, a sociedade, que tece novos laços de solidariedade e ação social.

Os princípios básicos da Reforma Sanitária seguem o padrão de BemEstar Social - a universalização do acesso aos serviços de saúde com equidade, a descentralização da gestão do sistema e dos serviços de saúde e a participação social. Um balanço da política de saúde mostra uma reformulação de prioridades do Ministério da Saúde durante os anos 90.

A expansão da oferta de ações e serviços relacionados à atenção básica ocorre, sobretudo em pequenas localidades, onde, até a metade da década de 90, a população ali residente dispunha de pouco ou nenhum acesso a bens e serviços públicos de saúde. Sem dúvida, expandir a cobertura é universalizar, mas a expansão só atenderá a universalidade e a equidade se levar em conta as necessidades sociais observadas.

A priorização de ações de saúde para grupos ditos vulneráveis promove uma intervenção que associa pobreza e saúde, fenômenos indissociáveis, 
mas de naturezas distintas. Dissolve, ainda, numa única intervenção racionalizadora, ações estigmatizadoras e focalizadas, que não consubstanciam um projeto unificado de proteção social para satisfazer exigências mínimas de renda, trabalho, acesso à saúde, educação e saneamento, qualificadas em sua diversidade regional e local.

A partir de 1995, com a formulação do Plano de Ações e Metas Prioritárias, o Ministério da Saúde afirma a Saúde da Família como estratégia política e promove incentivos para o Programa de Saúde da Família (PSF) e a abertura de contas específicas para o Piso de Atenção Básica (PAB), dissociadas da conta destinada à assistência médica, onde estão incluídos os repasses a título de pagamentos do custeio ambulatorial e hospitalar.

Essa política de descentralização da gestão e repasses de recursos entre esferas de governos é conduzida pela Norma Operacional Básica de 1996, que define formas mais complexas de gestão e financiamento do sistema, e, mais recentemente, pela Norma Operacional da Assistência à Saúde de 2001, que avança em torno da proposta de regionalização. Ainda são desconhecidos os resultados desta última e, particularmente, os ganhos obtidos em termos da melhoria das condições de vida da população gerados pela elevação de dispêndios em custeio dirigidos à média e alta complexidade da atenção, com base na concertação entre gestores.

Desde 1991, a emergência do Programa de Agentes Comunitários de Saúde (Pacs) no plano nacional se ajusta à política social do governo federal, que responde às exigências dos organismos internacionais no sentido da redução do papel do Estado, da focalização da atenção para população carente e da articulação entre serviços sociais e de saúde e a comunidade. Por esta percepção, o Pacs, responde, na esfera de governo federal, por uma ampliação do acesso a ações de promoção da saúde, que já se desenvolviam no âmbito de alguns governos estaduais ou de outras instituições da sociedade civil, com base no recrutamento de agentes sociais nas comunidades.

A percepção mais generalizada é que essa política de saúde é regressiva, caracterizada por um Estado assistencial, que responsabiliza a sociedade pela consecução do bem-estar, apoiando-se na filantropia, no voluntariado ou em trabalho simplificado do ponto de vista da atenção oferecida e da tecnologia disponível.

A inserção dos agentes comunitários de saúde (ACS) na rede do Sistema Único de Saúde (SUS) é ainda percebida como uma forma complementar de responder à necessidade de criar emprego e renda para as populações excluídas da política social, independente da afirmação de seus direitos sociais.

O Estado de Bem-Estar Social, em oposição ao que a política social dos governos aponta, é singular em estabelecer uma relação entre o direito conferido ao cidadão e o dever do Estado em fornecer recursos que o garantam. Esta forma de integração social implica a inclusão de recursos humanos ade- 
quados às condições e vida das pessoas e necessários à promoção do bemestar e que, por sua vez, são também pessoas portadoras de direitos.

Os ACS passam a estar a serviço do Estado e em condições de trabalho similares às dos funcionários públicos, sem, inicialmente, fazerem jus ao conjunto de direitos que a condição de servidor implica. Sua inserção é precária, informal e com baixa remuneração. Sua atividade permanente ao longo dos anos 90 e a incorporação a outros programas, como o PSF, abrem o debate sobre sua vinculação institucional, ou sobre a forma mais adequada de relação de trabalho para dar mais sustentabilidade ao uso desse recurso humano (Nogueira, Silva e Ramos, 2000).

A incorporação dos ACS às unidades básicas de saúde e, posteriormente, às equipes de Saúde da Família, de caráter multiprofissional, representa uma intervenção concreta ou um modo diferenciado de ação dentro do modelo de atenção à saúde e no debate a respeito de sua mudança. Os dois programas - Pacs e PSF — desenvolvidos segundo a lógica de seletividade de clientela dentro do SUS, ao se expandirem das comunidades rurais e periurbanas do Norte e Nordeste para grandes centros urbanos, mesmo que implantados em bairros onde há maior concentração de pobreza, aprofundam a experiência de focalização em um sistema que é universalista (Viana e Dal Poz, 1998).

Como se vê, ao longo do processo ampliam-se as condições favoráveis ao reconhecimento da identidade profissional dos ACS e de seus direitos trabalhistas e sociais, e abre-se espaço para que, em 2001, a implementação do Programa de Expansão da Estratégia de Saúde da Família (Proesf) para as grandes cidades, com mais de cem mil habitantes se faça a partir de outros patamares.

\section{ACS: uma nova profissão?}

A assertiva de que a profissionalização de uma nova atividade em saúde torna-se um fato social, sempre que surgem projetos de renovação das práticas sociais, significa que a introdução de um novo agente social na trama social responde às necessidades de uma nova ação. Assim, a emergência de um novo agente geralmente antecede à profissionalização, entendida como formação profissional e estabelecimento de diretrizes para sua realização, e a regulação dessa atividade a partir de um dado perfil profissional.

Todo processo de mudança na forma de organizar os procedimentos de produção dos serviços implica ajuste na formação do trabalhador, que deve ser reconhecido e valorizado, para que realmente se deflagre um processo de reorganização da força de trabalho em saúde e contribua decisivamente para a efetivação da política nacional de saúde. 
O Pacs tem em sua concepção os objetivos de oferecer uma resposta à ineficácia dos serviços públicos de saúde em combater as doenças já instaladas e estabelecer um vínculo entre o serviço e a população assistida. Ao atuar nas brechas deixadas pela baixa cobertura de ações básicas de saúde dentro e fora da unidade básica de saúde (UBS), atingindo populações isoladas, sem informação sobre cuidados com a saúde e colhendo informações sobre seu estado de saúde, os ACS imprimem uma nova dinâmica de atuação.

A implantação do PSF e a incorporação dos ACS neste programa reafirmam, nas UBS, a busca de estratégias para desenvolver uma atenção integral à saúde de indivíduos, grupos e famílias, que intervenham sobre fatores de risco aos quais a população está exposta e promovam parcerias com a sociedade, por meio de ações intersetoriais e estímulo do controle social. Ambos os programas se propõem a ter, como eixo de suas ações sociais e de saúde, a família e a comunidade, entendida como espaço social em que vivem e convivem indivíduos e famílias.

O agente comunitário de saúde é definido, neste novo contexto, como aquele profissional que atua no apoio aos indivíduos e coletivos sociais, por meio de atividades de promoção da saúde e prevenção de agravos, assim como de ações educativas e de acompanhamento a indivíduos, famílias e grupos, na mobilização de práticas de promoção da vida em coletividade e no desenvolvimento de interações sociais. Ele é um profissional "que pertence ao grupo de enfermagem, em virtude de realizar cuidados de saúde para com as pessoas" (Nogueira, Silva e Ramos, 2000, p. 5), embora para tal necessite de ampliar suas competências nesta área.

Ao mesmo tempo, é apresentado como o elo entre o serviço ou equipe e a comunidade, tendo como singularidade seu pertencimento à comunidade como forma de garantir a vinculação e a identidade cultural de grupo com as famílias sob sua responsabilidade. Neste sentido, ele é "um trabalhador genérico e fora de comum, não tendo similar entre as tradicionais ocupações e profissões da Saúde" (Nogueira, Silva e Ramos, 2000, p. 5), suas competências se desenvolvem no treinamento oferecido, independente de qualificação em profissão específica da saúde.

Essas interpretações sobre quem é, o que faz o ACS, segundo Nogueira, Silva e Ramos (2000), exercem forte pressão no sentido de identificar as ações que lhe podem ser delegadas e a qualificação formal necessária ao seu exercício profissional. Para as entidades de enfermagem, é importante distinguir o ACS do grupo de enfermagem, sendo mais fácil aceitá-lo como trabalhador genérico que atua na interação social entre serviço e comunidade.

Para sua incorporação à equipe de saúde, no primeiro momento, o ACS deve possuir competências básicas que são adquiridas no ensino fundamental, suficientes ao conhecimento e exercício da cidadania. 
O nível de ação do ACS exige, por um lado, a utilização de tecnologia simplificada no controle da doença, que envolve os fundamentos elementares de leitura e escrita, e o raciocínio aritmético para tratar de medidas. Por outro, exige um conhecimento bastante complexo para acompanhar indivíduos e grupos, no âmbito da identificação de risco ambiental, da promoção da saúde e da prevenção de doenças prevalentes. Esta combinação indica a emergência de uma profissão e a necessidade de uma melhor qualificação profissional, ou seja, a introdução de competências profissionais.

A melhoria da qualidade de vida da coletividade depende de ações de saúde tanto quanto de ações intersetoriais, que se espera sejam desenvolvidas pelo ACS. Este deve ser habilitado a identificar problemas na comunidade que têm impacto sobre a saúde e o bem-estar e a conhecer os recursos necessários para sustentar sua ação e atuação, de forma criativa e autônoma.

A autonomia que se espera do ACS na área social é relativa a sua capacidade de se autoplanejar, de gerenciar seu tempo e espaço de trabalho, de exercitar a criatividade e de participar em fóruns sociais onde se debatem os problemas da comunidade e aí influenciar a tomada de decisões. Esta autonomia também se expressa no trabalho em equipe, na interação com os usuários dos serviços em seus domicílios e no desenvolvimento da consciência da qualidade e das implicações éticas do trabalho, no planejamento de ações, na promoção da saúde, na prevenção da doença e no acompanhamento e avaliação das ações de saúde.

Ou seja, requer-se certa margem de liberdade e flexibilidade do agente em face de situações complexas e adversas que marcam sua ação e que exigem uma atuação competente na obtenção de determinado resultado. O sujeito-agente deve interagir para expandir o projeto técnico para o qual foi capacitado e transformá-lo num projeto comum da comunidade em resposta às necessidades de saúde (Peduzzi, 2001).

Esses atributos combinados compõem o que se denomina competência profissional do ACS, que supera a formação básica do indivíduo adquirida nas instituições de socialização, como família, escola e outras, e se desenvolvem ao longo do período por uma política explícita de educação profissional, pela organização de programa específico e eficaz de qualificação, que prepare os profissionais para atuar no sistema e na vida em geral (MS, 1999). Neste plano, também transcende o campo da saúde, pois requer aprendizagem em múltiplos aspectos das condições de vida da população.

Alguns passos oficiais indicam um avanço na trajetória da regulação da atividade profissional do ACS, conferindo-lhe o estatuto de uma nova profissão. Assim, o Decreto Federal no 3189/99 fixa as diretrizes para o exercício de atividades do ACS e fundamenta a posterior elaboração do projeto de lei que cria a profissão. A Lei no 10.507, aprovada em 10 de julho de 2002, que cria a profissão de Agente Comunitário de Saúde, explicita que o SUS é o âmbito de 
atuação deste profissional e estabelece novos requisitos mínimos para o exercício da profissão: ser residente na área de atuação, comprovar a conclusão do ensino fundamental e apresentar resultado satisfatório no curso de qualificação básica para a formação de ACS. Estabelece, ainda, que o ACS que já atuava como tal na data da publicação da lei assim é reconhecido profissionalmente, sendo dispensado de ter o ensino fundamental concluído.

A profissionalização do ACS, estimulada com a implantação e expansão do PSF, altera positivamente os perfis profissionais dos trabalhadores da saúde, ou seja, eleva o nível de escolarização e proporciona a esses profissionais, através da capacitação e educação permanente, a aquisição de uma compreensão global do processo produtivo pela apreensão de saber tecnológico, pela valorização da cultura do trabalho e pelo estímulo ao exercício da cidadania. Contudo, não regulamenta a formação profissional, além de deixar em aberto a questão do vínculo institucional e da remuneração.

\section{Formação profissional}

As normas legais reconhecem a profissão de ACS e estabelecem as diretrizes para a formação deste profissional. Esse processo de profissionalização foi conduzido pelo Ministério da Saúde durante a década de 90 e, segundo o Sistema de Informação da Atenção Básica do Ministério da Saúde (Siab), o resultado alcançado é, em parte, o esperado: o grau de escolaridade de $60 \%$ desses trabalhadores é mais elevado que a exigência legal; $18 \%$ deles têm apenas o ensino fundamental completo e $22 \%$ ainda não concluíram o ensino fundamental.

Com isto, apresentam-se novos desafios para os formuladores do programa de educação profissional para o ACS, visto o perfil singular desse trabalhador e as competências profissionais a serem desenvolvidas para o mesmo, que superam as exigências tradicionais ao setor saúde. Conforme destacam Silva e Dalmaso (2002), o setor saúde ainda não desenvolveu um saber sistematizado e instrumentos de trabalho e gerência sobre abordagem de família, sobre o contato com as situações de vida precária que determinam condições de saúde, sobre a desigualdade social e sobre a busca de cidadania, que tragam definições claras para o processo formador.

Assim, diante da ausência de um saber instituído, o profissional ACS age em função de sua experiência de vida e do seu perfil social, que guarda identidade com o grupo/comunidade a que pertence. Esses fatos não são generalizáveis, já que cada comunidade tem uma cultura própria, tem uma singular trajetória de ocupação do território e de organização solidária.

Pode-se compor o perfil do ACS local/municipal a partir de alguns indicadores como sexo, idade, escolaridade e escolarização, motivação para 
escolha da ocupação e percepções sobre seu processo de trabalho e desenvolvimento de atividades, como os projetados pelo estudo encomendado pelo Ministério da Saúde.

Este estudo com objetivos bem definidos3, voltados para a avaliação do processo de implementação do PSF em grandes centros urbanos, reflete sobre a profissionalização dos ACS e como essa tem correspondido ao propósito de mudar o modelo de atenção. Assim, seguem-se alguns comentários.

Analisando o perfil dos profissionais das equipes de saúde da família (ESF) estudadas, observa-se entre os ACS escolaridade alta em relação às exigências do cargo acordadas entre o Ministério da Saúde e as secretarias municipais de saúde - saber ler e escrever. Em cinco dos municípios, os percentuais de ACS com segundo grau completo superam a faixa de $60 \%$, embora apenas um percentual pequeno tenha curso técnico em saúde.

Em relação à remuneração, o salário dos profissionais da saúde da família varia bastante. Em alguns municípios estudados, os ACS recebem salário mínimo, embora esta não seja a regra. Observa-se, na maioria dos integrantes da ESF certa insatisfação salarial, pois não se sentem bem remunerados, considerando o conjunto das suas atribuições dentro do programa.

Exige-se para a inserção do ACS no PSF tempo integral, o que pode determinar a predominância feminina e de jovens no PSF e reforçar a não progressividade porque não garante a ascensão social nem favorece a continuidade dos estudos. Além disso, constata-se que a maioria deles, em diversos municípios, escolheu atuar no PSF por estar em situação de desemprego, embora não veja o programa como um mercado de trabalho promissor, pela renda auferida e por não oferecer condições de ascensão.

$\mathrm{O}$ fato de trabalhar com comunidades pobres é um fator predominante entre $55 \%$ ou mais ACS em todos os casos estudados, o que é decorrente do critério de seleção adotado para este profissional no PSF — ser residente na comunidade há mais de dois anos - , associado ao fato de que, na grande maioria dos municípios, a escolha das áreas de implantação do programa se inicia pela definição de áreas com uma situação de vulnerabilidade maior, daí se expandindo para o resto do território.

Um fator facilitador para a implementação do PSF é a adesão do conjunto dos profissionais à dimensão ideológica do programa e a percepção de que o trabalho tem uma dimensão social, que se expressa em solidariedade social.

A percepção dos ACS quanto ao investimento em capacitação em saúde da família é especialmente elevada, mais de $70 \%$ declarou ter recebido o treinamento introdutório, capacitação mínima para o exercício profissional, prescrita pelo Ministério da Saúde. A capacitação também é percebida pelos gestores como uma das alavancas da proposta de mudança, da reorganização do modelo de atenção básica, porque provoca nos profissionais do PSF a revisão dos seus conhecimentos em relação à prática. 
Os gestores destacam a importância dos treinamentos introdutórios especialmente voltados para os ACS, dado sua inovação no campo dos cuidados e no campo da promoção social. Contudo, apontam que a capacitação inicial ou educação permanente dos ACS não deve se limitar a reproduzir a normativa do Ministério da Saúde e deve enfatizar o debate do modelo local implantado.

As principais dificuldades, entretanto, se expressam na pouca participação dos pólos de capacitação das equipes de saúde da família na educação permanente dos profissionais em diversos centros urbanos, que nem sempre cumprem sua principal atribuição, que é oferecer os treinamentos introdutórios. Estes ficam a cargo de núcleos de desenvolvimento técnico dos municípios, que estabelecem diferentes prioridades, exigindo um esforço de articulação das secretarias municipais com as instituições formadoras e de representação de interesse, como os conselhos regionais e associações corporativas, para promover a capacitação necessária aos profissionais do PSF.

Uma singularidade dos profissionais da saúde da família é atuar no âmbito da intersetorialidade, ou seja, na dimensão de uma área social que faz interface entre saúde e outras políticas sociais, como assistência social, educação, trabalho, nutrição, saneamento e meio ambiente, que são responsáveis pela garantia às pessoas e à coletividade de condições de bem-estar físico, mental e social, que determinam o estado de saúde da população (MS, 2003, p. 26).

Neste aspecto, nota-se que a realização de atividades extra-setoriais pelos integrantes da ESF é um importante indicador da prioridade para atividades de promoção da saúde. No campo da saúde, o ACS enfrenta situações que exigem ação responsável, com iniciativa, discernimento e objetividade na percepção dos fatos que afetam a saúde da população, além de habilidade para interagir com outros atores e mobilizá-los para uma ação integrada.

Tal competência possibilita ao trabalhador conhecer a utilidade e os impactos das ações que realiza, compreender que os grupos sociais não são abstratos ou distantes, conhecendo suas necessidades e modos de viver, e, sobretudo, compreender a importância do processo de interação da equipe de trabalho com os indivíduos, grupos e coletividades com os quais atua.

A atuação intersetorial é investigada, considerando-se a percepção dos profissionais quanto à realização de atividades junto a outros órgãos de políticas públicas pelos profissionais integrantes das ESF, e a avaliação das famílias quanto ao conhecimento da ESF sobre os problemas de saúde vividos pela comunidade.

Assim, a maioria dos ACS participou nos últimos seis meses anteriores à pesquisa de "atividades para a solução/encaminhamento de problemas de sua comunidade junto a outros órgãos públicos ou entidades da sociedade nas cidades estudadas", e identificaram-se como principais problemas tra- 
tados pela comunidade, em ordem de prioridade: a coleta de lixo, a escola/educação, o abastecimento de água e o esgoto (Escorel et al., 2002, p. 139).

Os ACS indicam percepções positivas quanto à ação intersetorial das equipes de saúde da família (ESF) e concordaram muito com a afirmativa: “o PSF favorece a ação intersetorial no município" (Escorel et al., 2002, p. 140).

Outro aspecto singular investigado diz respeito ao trabalho em equipe, levantando as percepções dos profissionais sobre o perfil das ESF quanto ao relacionamento, à priorização de atividades, rotinas e procedimentos. Estes dados servem para analisar como as competências profissionais definidas para os ACS são observadas em sua prática cotidiana e representam uma nova perspectiva de organização dos processos de formação e de trabalho de equipe.

Os membros da ESF avaliam positivamente seu trabalho em equipe. Suas percepções sobre a organização da equipe são positivas, reconhecendo que a equipe "é sensível e aberta a mudanças, com capacidade de rever rotinas" e tem um "relacionamento bom e respeitoso". Em oposição foi pequeno o índice ( $2 \%$ ) de profissionais que apontavam na ESF "um relacionamento conflituoso" (Escorel et al., 2002, p. 165).

No que diz respeito ao processo de trabalho, o grau de participação e discussão da programação das atividades da ESF com regularidade semanal ou quinzenal também se mostrou elevado no conjunto das equipes municipais com freqüência superior a 50\% em todos os seis casos estudados (Escorel et al., 2002).

As atividades extramuros realizadas por ACS das ESF são expressão de uma relação entre atividades assistenciais e atividades promocionais junto à comunidade, componentes do novo perfil de atenção. Pode-se afirmar que as atividades de campo junto à comunidade têm grande adesão no conjunto de profissionais e constata-se que as visitas domiciliares foram realizadas em elevadas proporções por diversos os profissionais da ESF, na semana anterior à pesquisa.

A grande maioria dos ACS dos casos estudados declarou realizar visitas domiciliares com freqüência que varia principalmente em relação ao número de famílias adscritas a cada equipe. No mínimo, fazem uma visita mensal a cada família, mas a freqüência aumenta quando o número de famílias sob a responsabilidade dos ACS é menor. A variação do número de famílias sob a responsabilidade dos ACS é uma decisão política da coordenação municipal do programa, em função de sua estratégia de extensão da cobertura das UBS ou ESF.

Analisando-se as atividades não assistenciais realizadas na semana anterior à pesquisa por integrantes da ESF, pode-se conhecer aspectos da dinâmica do trabalho em equipe. Observa-se que os profissionais de nível superior são responsáveis pela supervisão direta das atividades de auxiliares de enfermagem e ACS. Em metade dos municípios, essas atividades estão a 
cargo dos enfermeiros, indicando a interlocução muito próxima entre o grupo de enfermagem e o ACS.

Outras atividades não assistenciais de administração e gerência são desenvolvidas em pequenas proporções pelos profissionais da ESF, sendo mais freqüentes entre os profissionais de nível superior (médicos e enfermeiros). Contudo, a maioria dos profissionais das ESF, entre os quais $65 \%$ dos ACS, assinalaram que participou de reunião de equipe na semana anterior a pesquisa. A reunião de equipe é entendida como instrumento de trabalho fundamental ao desenvolvimento de uma nova prática social em saúde, é o momento de troca social do coletivo em torno da programação de atividades e da exposição de dificuldades no cotidiano do trabalho.

Essas observações destacadas dentro de um estudo mais amplo indicam que o ACS está incorporado nas ESF, desenvolvendo o conjunto de atributos sociais que lhe é imputado, e exercendo o papel de mediador entre a comunidade que representa e cuida e a unidade de saúde, por expressar dentro dela os valores culturais que compartilha com o grupo. Mais do que isso, ao desempenhar as atividades intersetoriais, articula os objetivos das políticas sociais do Estado e os valores próprios da comunidade.

\section{Considerações finais}

O Pacs e o PSF têm tido papel preponderante no cenário das políticas de saúde no Brasil, no sentido da reorganização da atenção básica na década de 90. A inserção dos ACS como um novo trabalhador de saúde tem impacto no debate e no desenho da mudança do modelo de atenção à saúde e na definição do cuidado em saúde.

O contexto em que é implementado amplia sua dimensão para o campo da política de proteção social em saúde, ao dar prioridade ao trabalho intersetorial e à esfera da promoção da saúde. Inova ao buscar um novo agente que articula identidade comunitária com solidariedade social, mesmo que esta se dê em forma mais orgânica, em conformidade com o desenvolvimento social no Brasil.

Esse agente desempenha um papel de mediação, que ativa a interlocução dos atores sociais quanto aos seus valores éticos, suas necessidades, suas formas de conhecimento e capacidade de constituir rede de apoio social e garantia de direitos.

A atuação do ACS hoje é limitada por uma socialização tradicional, mas aponta para a necessidade de se redimensionar o status que o setor saúde tem dentro das políticas setoriais, ao implementar sua reforma, renovar suas prioridades e investir na reorganização do processo de trabalho em saúde, onde saúde tem uma definição ampliada como direito social. 


\section{Notas}

1 Pesquisadora titular da Escola Nacional de Saúde Pública, Fiocruz. Doutora em Saúde Coletiva.<mhelenam@ensp.fiocruz.br>.

2 O pacto firmado na Constituição Federal de 1988 não se estabeleceu, de fato, numa política integrada entre previdência, saúde e assistência. No âmbito institucional-legal, foram promulgadas leis específicas para cada área - a Lei Orgânica da Saúde (Lei 8080 e 8142/90), a Lei Orgânica da Seguridade Social (Lei 8212/91 — de fato regulando a previdência social) e a Lei Orgânica da Assistência Social (Lei 8742/93). Cada uma das áreas definiu ações e programas específicos e isolados para o desenvolvimento de suas políticas, disputando por recursos e sem dar forma à política de seguridade social.

3 A pesquisa "Avaliação da implantação do PSF em grandes centros urbanos: dez estudos de caso", coordenada por Sarah Escorel, Ligia Giovanella, Maria Helena Mendonça, Rosana Magalhães e Monica Senna, tinha como objetivo analisar os fatores facilitadores e limitantes do processo de implementação do PSF nos municípios de Aracaju (SE), Camaragibe (PE), Campinas (SP), Goiânia (GO), Manaus (AM), Palmas (TO), São Gonçalo(RJ), Vitória (ES), Vitória da Conquista (BA) e Brasília(DF), quanto ao estabelecimento de vínculos entre ESF e comunidade, à conversão do modelo de atenção à saúde nas unidades básicas e sua articulação à rede de serviços de saúde. 


\section{Referências}

ESCOREL, Sarah et al., 2002. Avaliação da implantação do programa saúde da familia em dez grandes centros urbanos: sintese dos principais resultados. Brasília, DF: MS/Secretaria de Políticas de Saúde/Departamento de Atenção Básica.

MENDONÇA, Maria Helena M. 2002. Sistema Único de Saúde no Brasil: entre o formal e o real: as vicissitudes da construção de uma intervenção pública moderna. Cebes, Revista Saúde em Debate, n. 54, p. $9-24$

MERHY, Emerson; FRANCO, Thulio, 1999. PSF: contradições de um programa destinado à mudança do modelo tecnoassistencial. São Paulo: Unicamp. S/l, (Mimeo).

MS (MINISTÉRIO DA SAÚDE). CONSELHO NACIONAL DE SAÚDE. 2003. 2. ed. Princípios e diretrizes para a $\mathrm{NOB} / \mathrm{RH}-$ SUS. Brasília: Editora do Ministério da Saúde.

MS (MINISTÉRIO DA SAÚDE). 1999. Diretrizes para elaboração de programas de qualificação e requalificação dos ACS -
Desenvolvimento Humano. Brasília, DF: Secretaria de políticas de saúde. Coordenação geral de desenvolvimento de recursos humanos para o SUS. (Mimeo). NOGUEIRA, Roberto P; SILVA, Frederico B, RAMOS, Zuleide, V., 2000. A vinculação institucional de um trabalhador sui generis: o agente comunitário de saúde. Rio de Janeiro: Ipea. Textos para Discussão n. 735.

PEDUZZI, M. 2001. Equipe multiprofissional de saúde: conceito e tipologia. $R e-$ vista de Saúde Pública, v. 35, n. 1, p. 103-109.

SILVA, Joana. A.; DALMASO, Ana Silvia W. 2002. Agente comunitário de saúde: o ser, o saber, o fazer. Rio de Janeiro: Fiocruz.

VIANA, A. L.; DAL POZ, M. 1998. A reforma do sistema de saúde no Brasil e o programa de Saúde da Família. Physis: Revista de Saúde Coletiva, v. 8, n. 2. p.11-48. 